\title{
Site-Specific Manipulation of CNTs to EXpressLO'M Grids for TEM Analysis
}

\author{
Paul A. Anzalone ${ }^{1}$ and Lucille A. Giannuzzi ${ }^{2}$ \\ ${ }^{1}$ Nanocomp Technologies, Inc., Merrimack, NH USA. \\ 2.EXpressLO LLC, Lehigh Acres, FL USA.
}

Carbon nanotubes (CNTs) may be prepared for transmission electron microscopy (TEM) by processing them in a liquid solution and then dispersing them onto holey carbon coated TEM grids [1]. However, residual solution products remaining on the CNTs may alter their surface structure. In addition, the carbon thin film may inhibit analysis of the CNTs, and it is difficult to prevent agglomeration and force individual CNTs to bridge across open holes in the film so they can be viewed directly through vacuum (e.g., without viewing through a support film). In addition, methods of isolating $2 \mathrm{D}$ graphene from graphite via micromechanical cleaving with adhesive tape [2] may damage multi-walled CNTs surfaces or CNT ends. The authors have vast experience in focused ion beam (FIB) specimen preparation methods but prefer avoiding possible damage to the CNTs by ion implantation or recoil displacement.

Thus, in this paper we use an ex situ lift out micromanipulator system to pick up CNTs from site specific plies of sheet material and manipulate them across an open slotted EXpressLO ${ }^{\mathrm{TM}}$ grid carrier [3], using glue to facilitate the adhesion, in a manner similar to that previously performed for particles [4]. The advantages to the Pick\&Place ${ }^{\mathrm{TM}}$ method are numerous: (i) it does not damage the CNTs under investigation, (ii) it is fast and easy, (iii) it is site specific, and (iv) the CNTs may be viewed directly in vacuum and not through a support film.

Figure 1 shows light optical micrographs of the Pick\&Place ${ }^{\mathrm{TM}}$ process. In figure 1a the glass probe is dipped into a drop of M-Bond adhesive glue. The glue is then painted onto the grid support arms on both sides of the open slotted grid carrier (figure 1b). A clean glass probe is then used to pick up several CNTs from a site specific region (e.g., spatially to within $\sim 1 \mu \mathrm{m}$ ) in a ply of sheet material mounted in crosssection (figures 1c,d). The CNTs are then manipulated across an open grid slot (figure 1d) and the probe may be used to lightly tamp the end of the CNTs onto the glue (figure 1e). Note the ends pulled from the ply are glued to the grid, so undamaged CNT material centered across the open slot is observed. The entire process takes only a few minutes and may be viewed in the YouTube movie in reference [5]. The process may be repeated multiple times to position CNTs from the same or different region or ply across numerous open slots in the grid carrier. It is recommended to use a new clean probe tip each time a new region of CNTs are picked up to prevent cross-contamination of the CNTs. The glue may be cured by heating for a few minutes as per manufacturer guidelines or in ambient conditions for longer times if heating to high temperatures is a concern. It some cases, the glue may not even be necessary.

Figure 2 shows $80 \mathrm{keV}$ bright field TEM images of the CNTs. In the low magnification image in figure $2 \mathrm{a}$, the CNTs are observed spanning the open slot of the EXpressLO ${ }^{\mathrm{TM}}$ grid. The higher magnification image in figure $2 \mathrm{~b}$ shows the mostly single walled structure of these CNTs. This Pick\&Place ${ }^{\text {TM }}$ manipulation TEM specimen preparation technique allows for easy and site-specific preparation without damage to the CTNs or interference from a carbon support film [6]. 


\section{References:}

[1] J.E. Decker et al., Metrologia 46 (2009) p. 682.

[2] K.S. Novoselov, et al., Science 306, (2004) p. 666.

[3] L.A. Giannuzzi et al., Microsc. Microanal., 21 (2015) p.1032.

[4] L.A. Giannuzzi and F.A. Stevie (eds.) Intro. to Focused Ion Beams, (2005) Springer, 222-223.

[5] www.YouTube.com/LAGiannuzzi/videos

[6] TEM images were obtained using an FEI Talos F200X at The Pennsylvania State University MCL.



Figure 1. Light optical micrographs of the Pick\&Place ${ }^{\mathrm{TM}}$ manipulation process for CNTs. (a) dabbing the probe into glue, (b) placing glue on the EXpressLO ${ }^{\mathrm{TM}}$ support grid using the probe, (c,d) picking up CNTs with a clean probe, (e) positioning CNTs across an open slot, (e) tamping the CNTs into the glue with the probe. For reference, the tip probe diameter is $\sim 1 \mu \mathrm{m}$.

(a)

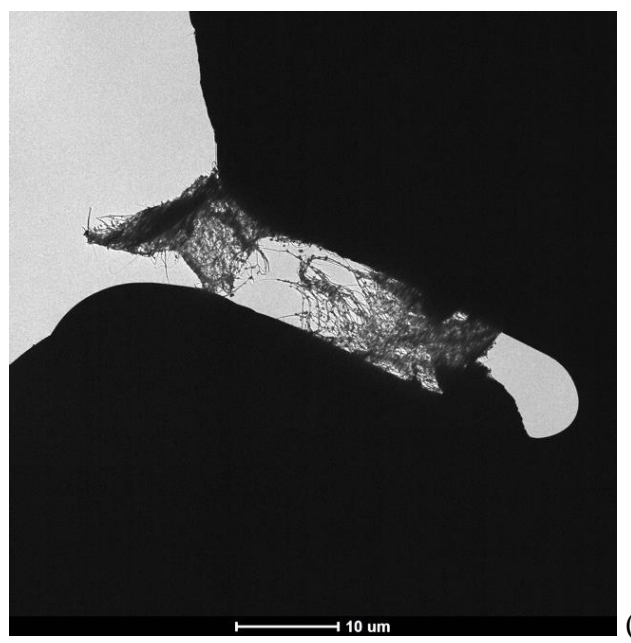

(b)

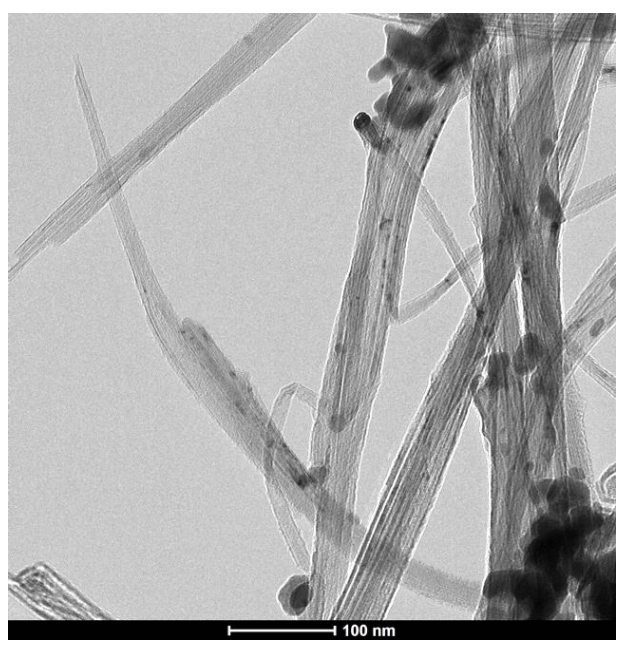

Figure 2. $80 \mathrm{keV}$ Bright field TEM images of CNTs viewed with no carbon support film. (a) low magnification image and (b) high magnification image. 